\title{
Estimates of character sums with exponential function
}

\author{
by \\ Hong Bing Yu (Hefei)
}

1. Introduction. In [3] Dobrowolski and Williams proved, among other things, the following interesting result:

$$
\left|\sum_{x=1}^{X}\left(\frac{a g^{x}+b}{p}\right)\right| \leq \frac{\sqrt{p-1} \log (p-1)}{2 \log 2}+3 \sqrt{p-1},
$$

where $p$ is an odd prime, $g$ is a primitive root $\bmod p,\left(\frac{x}{p}\right)$ is the Legendre symbol, and $a, b, X$ are integers satisfying $p \nmid a b$ and $1 \leq X \leq p-1$.

The purpose of this paper is to extend (1.1) in two directions. We first prove the following result, which may be compared with the classical inequality of Pólya-Vinogradov for character sums with linear polynomial (cf. Davenport $[2, \S 23])$.

TheOREM 1. Let $n \geq 2$ and $\lambda$ be integers with $(n, \lambda)=1$ and $\lambda$ belonging to the exponent $d$ modulo $n$. Let $\chi$ be a primitive Dirichlet character modulo n. Write

$$
S_{n}(\chi, \lambda, X)=\sum_{x=1}^{X} \chi\left(a \lambda^{x}+b\right),
$$

where $a, b$ and $X$ are integers satisfying $(a b, n)=1$ and $1 \leq X \leq d$. Then

$$
\left|S_{n}(\chi, \lambda, X)\right|<\sqrt{n}\left(\frac{2}{\pi} \log n+\frac{7}{5}\right) .
$$

We note that for an imprimitive $\chi$ no non-trivial bound for $\left|S_{n}(\chi, \lambda, X)\right|$ can be obtained. Consider, for instance, the case where $n=p^{k}$ ( $p$ odd prime and $k \geq 2$ ) and $\chi$ being induced by a (primitive) character modulo $p$. Let $\lambda \equiv 1(\bmod p), \lambda \not \equiv 1\left(\bmod p^{2}\right), a+b \equiv 1(\bmod p)$ and $p \nmid a b$. Then it is easily seen that $\lambda$ belongs to exponent $p^{k-1}$ modulo $n$ and that $S_{n}(\chi, \lambda, X)=X$ for $X \geq 1$.

2000 Mathematics Subject Classification: Primary 11L40.

Project supported by the National Natural Science Foundation of China. 
Furthermore, we show that in certain cases the inequality (1.3) is essentially best possible.

Theorem 2. Let $n=p^{k}, p$ an odd prime, $k \geq 2$. Let $\chi$ be a primitive character modulo $n, g$ be a primitive root modulo $n$, and $a, b$ be integers with $p \nmid a b$. Let $S_{n}(\chi, g, X)$ be as in (1.2). Then

$$
\max _{1 \leq X \leq \varphi(n)}\left|S_{n}(\chi, g, X)\right| \gg \sqrt{n}
$$

where the implicit constant is absolute.

Our second purpose is to generalize (1.1) to general finite fields. As usual, let $F_{q}$ denote the finite field of $q=p^{k}$ elements ( $p$ prime and $k \geq 1$ ), and let $F_{q}^{*}$ be the multiplicative group of non-zero elements of $F_{q}$. Let $\widehat{F_{q}^{*}}$ be the set of all multiplicative characters of $F_{q}^{*}$, and $\varepsilon \in \widehat{F_{q}^{*}}$ be the trivial character. For any $\psi \in \widehat{F_{q}^{*}}$ it is convenient to extend the definition of $\psi$ by setting $\psi(0)=0$.

Theorem 3. Let $\lambda \in F_{q}^{*}$ belong to the exponent d. Let $\chi \in \widehat{F_{q}^{*}}$ and $\chi \neq \varepsilon$. Write

$$
T_{q}(\chi, \lambda, X)=\sum_{x=1}^{X} \chi\left(a \lambda^{x}+b\right),
$$

where $a, b \in F_{q}^{*}, X$ is integer with $1 \leq X \leq d$. Then

$$
\left|T_{q}(\chi, \lambda, X)\right|<\sqrt{q}\left(\frac{2}{\pi} \log q+\frac{7}{5}\right) .
$$

By Theorem 3 and an argument similar to that used in Burgess $[1, \S 6]$, we have the following result which may be of independent interest.

Corollary. Let $g$ be a primitive root of $F_{q}$, and $a, b \in F_{q}^{*}$. For integers $X, Y$ with $1 \leq Y<Y+X \leq q-1$, we denote by $H(X, Y)$ the number of primitive roots in the set

$$
\left\{a g^{x}+b \mid Y \leq x \leq Y+X-1,(x, q-1)=1\right\} .
$$

Then

$$
H(X, Y)=\frac{\varphi(q-1)}{q-1}\left(\frac{\varphi(q-1)}{q-1} X+\theta 4^{\omega(q-1)} \sqrt{q} \log q\right),
$$

where $\omega(q-1)$ is the number of different prime divisors of $q-1$, and $|\theta| \leq 4$.

Finally, we show that the inequality (1.6) is also close to best possible.

THEOREM 4. Let $g$ be a primitive root of $F_{q}, a, b \in F_{q}^{*}$, and $T_{q}(\chi, \lambda, X)$ be as in (1.5). Then 


$$
\max _{1 \leq X \leq q-1}\left|T_{q}(\chi, g, X)\right| \gg \sqrt{q}
$$

where the implicit constant is absolute.

2. Proof of Theorem 1. Our argument is a modification of that used in the proof of the Pólya-Vinogradov inequality mentioned above.

Write $e_{n}(y)=e^{2 \pi i y / n}$ as usual. For any Dirichlet character $\psi$ modulo $n$, the Gaussian sum $G(\psi)$ is defined by

$$
G(\psi)=\sum_{y=1}^{n} \psi(y) e_{n}(y),
$$

where the accent indicates that we only consider those $y$ coprime to $n$.

For reference purposes, we state the following well known result in the form of a lemma (cf. Davenport $[2, \S 9]$ ).

Lemma 1. (i) If $(m, n)=1$, then

$$
\psi(m) G(\bar{\psi})=\sum_{y=1}^{n} \bar{\psi}(y) e_{n}(m y) .
$$

(ii) If $\psi$ is a primitive character modulo $n$, then (2.2) holds for any $m$.

(iii) For any character $\psi$ modulo $n$ we have $|G(\psi)| \leq \sqrt{n}$, and the equality holds if and only if $\psi$ is primitive.

We now prove Theorem 1. Since $\chi$ is a primitive character modulo $n$, we have by Lemma 1(ii) and (1.2),

$$
\begin{aligned}
S_{n}(\chi, \lambda, X) & =\frac{1}{G(\bar{\chi})} \sum_{y=1}^{n} \bar{\chi}(y) e_{n}(b y) \sum_{x=1}^{X} e_{n}\left(a y \lambda^{x}\right) \\
& =\frac{1}{G(\bar{\chi})} \sum_{y=1}^{n} \bar{\chi}(y) e_{n}(b y) S_{y}(X),
\end{aligned}
$$

say. Further, since $\left(a y \lambda^{x}, n\right)=1$, it follows that $e_{n}\left(a y \lambda^{x}\right)$ can be expanded into a finite Fourier series with respect to the Dirichlet characters $\psi$ modulo $n$. We have (using (2.1)), for $x=1, \ldots, X$,

$$
e_{n}\left(a y \lambda^{x}\right)=\frac{1}{\varphi(n)} \sum_{\psi} G(\bar{\psi}) \psi\left(a y \lambda^{x}\right)
$$

and so

$$
S_{y}(X)=\frac{1}{\varphi(n)} \sum_{\psi} G(\bar{\psi}) \psi(a y) \sum_{x=1}^{X} \psi\left(\lambda^{x}\right) .
$$


Substituting this in (2.3) and using Lemma 1(i) (noting that $(b, n)=1$ ), we get

$$
\begin{aligned}
S_{n}(\chi, \lambda, X) & =\frac{1}{G(\bar{\chi}) \varphi(n)} \sum_{\psi} G(\bar{\psi}) \psi(a) \sum_{y=1}^{n} \bar{\chi}(y) \psi(y) e_{n}(b y) \sum_{x=1}^{X} \psi\left(\lambda^{x}\right) \\
& =\frac{1}{G(\bar{\chi}) \varphi(n)} \sum_{\psi} G(\bar{\psi}) G(\bar{\chi} \psi) \psi(a) \chi \bar{\psi}(b) \sum_{x=1}^{X} \psi\left(\lambda^{x}\right) .
\end{aligned}
$$

Recall that $\lambda$ belongs to the exponent $d$ modulo $n$. Thus each $d$ th root of unity $e_{d}(j)(0 \leq j \leq d-1)$ occurs exactly $\varphi(n) / d$ times as a value of $\psi(\lambda)$. Therefore, by (2.4) and Lemma 1(iii), we have (cf. the proof of Niederreiter [6, Theorem 8.3])

$$
\begin{aligned}
\left|S_{n}(\chi, \lambda, X)\right| & \leq \frac{\sqrt{n}}{\varphi(n)} \sum_{\substack{\psi \\
\psi(\lambda) \neq 1}} \frac{2}{|1-\psi(\lambda)|}+\frac{X}{\varphi(n)} \cdot \frac{\varphi(n)}{d} \sqrt{n} \\
& =\frac{2 \sqrt{n}}{\varphi(n)} \cdot \frac{\varphi(n)}{d} \sum_{j=1}^{d-1} \frac{1}{\left|1-e_{d}(j)\right|}+\frac{X}{d} \sqrt{n} \\
& =\frac{\sqrt{n}}{d} \sum_{j=1}^{d-1} \frac{1}{|\sin (\pi j / d)|}+\frac{X}{d} \sqrt{n} \\
& \leq \frac{\sqrt{n}}{d}\left(\frac{2}{\pi} d \log d+\frac{2}{5} d\right)+\frac{X}{d} \sqrt{n},
\end{aligned}
$$

where we have used Lemma 2 of Niederreiter [5] in the last step. Theorem 1 then follows, since $X \leq d<n$.

3. Proof of Theorem 2. In this section we write $r=\varphi(n)$ and let

$$
S(x)=\sum_{0 \leq y \leq x} \chi\left(a g^{y}+b\right)
$$

for $x \geq 0$. The function $S(r x)$ is of bounded variation, and hence has a convergent Fourier series

$$
S(r x)=\sum_{m=-\infty}^{\infty} c_{m} e^{2 \pi i m x} \quad \text { for } 0<x<1 .
$$

Clearly

$$
c_{0}=\int_{0}^{1} S(r x) d x=\frac{1}{r} \sum_{l=0}^{r-1} S(l) .
$$


For $m \neq 0$, we have

$$
\begin{aligned}
c_{m} & =\int_{0}^{1} S(r x) e^{-2 \pi i m x} d x=\frac{1}{r} \int_{0}^{r} S(x) e_{r}(-m x) d x \\
& =\frac{1}{2 \pi i m}\left(\sum_{l=0}^{r-1} \chi\left(a g^{l}+b\right) e_{r}(-l m)-\sum_{l=0}^{r-1} \chi\left(a g^{l}+b\right)\right) .
\end{aligned}
$$

By the hypothesis of Theorem 2 it is easily seen that

$$
\begin{aligned}
\sum_{l=0}^{r-1} \chi\left(a g^{l}+b\right) & =\sum_{\substack{y=1 \\
p \nmid y}}^{p^{k}} \chi(y+b)=\sum_{\substack{y=1 \\
p \nmid y}}^{p^{k}} \chi(y)-\sum_{y=1}^{p^{k-1}} \chi(b+p y) \\
& =-\sum_{y=1}^{p^{k-1}} \chi(b+p y)=0
\end{aligned}
$$

(a proof of the last equality can be found in Davenport [2, p. 66]). Moreover, by the same argument used in Section 2 and using the same notation as there, we arrive at the identity

$$
\begin{aligned}
& \sum_{l=0}^{r-1} \chi\left(a g^{l}+b\right) e_{r}(-l m) \\
& \quad=\frac{1}{r G(\bar{\chi})} \sum_{\psi} G(\bar{\psi}) G(\bar{\chi} \psi) \psi(a) \chi \bar{\psi}(b) \sum_{l=0}^{r-1} \psi\left(g^{l}\right) e_{r}(-l m) .
\end{aligned}
$$

The inner sum is equal to $r$ if $\psi(g)=e_{r}(m)$ and equal to 0 otherwise. Clearly, $\psi(g)=e_{r}(m)$ if and only if $\psi=\psi_{m}$, where $\psi_{m}$ is the Dirichlet character modulo $n$ given by

$$
\psi_{m}(x)=e_{r}\left(s \operatorname{ind}_{g} x\right), \quad 0<s \leq r \text { and } s \equiv m(\bmod r) .
$$

Thus by (3.3)-(3.5) we have, for $m \neq 0$,

$$
c_{m}=\frac{1}{2 \pi i m G(\bar{\chi})} G\left(\bar{\psi}_{m}\right) G\left(\bar{\chi} \psi_{m}\right) \psi_{m}(a) \chi \bar{\psi}_{m}(b) .
$$

Since $\chi$ is primitive, we may write $\chi(x)=e_{r}\left(t\right.$ ind $\left._{g} x\right)$ with $0<t<r$ and $p \nmid t$. From (3.6) we see that if (and only if) $m$ satisfies $m \neq \equiv, t(\bmod p)$, then both $\psi_{m}$ and $\bar{\chi} \psi_{m}$ are primitive characters modulo $n$. Thus, by Lemma 1(iii) and (3.7), we have

$$
\left|c_{m}\right|=\frac{\sqrt{n}}{2 \pi|m|} \quad \text { for } m \not \equiv 0, t(\bmod p) .
$$

On applying Parseval's formula to (3.2) and using (3.8), we have 


$$
\frac{1}{r} \sum_{l=0}^{r-1}|S(l)|^{2}=\int_{0}^{1}|S(r x)|^{2} d x=\sum_{m}\left|c_{m}\right|^{2} \gg n .
$$

Now, in view of (1.2), (3.1) and (3.4), Theorem 2 follows immediately.

4. Proof of Theorems 3 and 4. We first prove Theorem 3. Our argument is a version of that used in Section 2. We observe that $\chi\left(a \lambda^{x}+b\right)$, considered as a function of $b$, can be expanded into a finite Fourier series with respect to the multiplicative characters of $F_{q}^{*}$. Thus, for $x=1, \ldots, X$,

$$
\chi\left(a \lambda^{x}+b\right)=\sum_{\psi} c_{\psi} \bar{\psi}(-b),
$$

with the Fourier coefficients

$$
c_{\psi}=\frac{1}{q-1} \sum_{y \in F_{q}^{*}} \chi\left(a \lambda^{x}+y\right) \psi(-y)=\frac{1}{q-1} \chi \psi\left(a \lambda^{x}\right) J(\chi, \psi)
$$

where $J(\chi, \psi)$ is a Jacobi sum

$$
J(\chi, \psi)=\sum_{u \in F_{q}} \chi(1+u) \psi(-u)
$$

(note that we have defined that $\psi(0)=0$ for any $\psi \in \widehat{F_{q}^{*}}$ ). By $(1.5),(4.1)$ and (4.2) we have

$$
\begin{aligned}
T_{q}(\chi, \lambda, X) & =\frac{1}{q-1} \sum_{\psi} J(\chi, \psi) \chi \psi(a) \bar{\psi}(-b) \sum_{x=1}^{X} \chi \psi\left(\lambda^{x}\right) \\
& =\frac{1}{q-1} \sum_{\psi} J(\chi, \bar{\chi} \psi) \psi(a) \chi \bar{\psi}(-b) \sum_{x=1}^{X} \psi\left(\lambda^{x}\right) .
\end{aligned}
$$

It is well known that

$$
|J(\chi, \bar{\chi} \psi)| \leq \sqrt{q} \quad \text { with equality if } \psi \neq \varepsilon, \chi
$$

(cf. Lidl and Niederreiter [4, Chapter 5]). Moreover, since $\lambda$ belongs to the exponent $d$, it follows that each $d$ th root of unity $e_{d}(j)(0 \leq j \leq d-1)$ occurs exactly $(q-1) / d$ times as a value of $\psi(\lambda)$. Hence, by $(4.3),(4.4)$ and the argument used in Section 2, we have

$$
\begin{aligned}
\left|T_{q}(\chi, \lambda, X)\right| & \leq \frac{\sqrt{q}}{q-1} \cdot \frac{q-1}{d} \sum_{j=1}^{d-1} \frac{1}{|\sin (\pi j / d)|}+\frac{X \sqrt{q}}{q-1} \cdot \frac{q-1}{d} \\
& <\sqrt{q}\left(\frac{2}{\pi} \log q+\frac{7}{5}\right) .
\end{aligned}
$$

This completes the proof of Theorem 3 . 
Proof of Theorem 4. We proceed as in the proof of Theorem 2. Write $r=q-1$ and let

$$
T(x)=\sum_{0 \leq y \leq x} \chi\left(a g^{y}+b\right)
$$

for $x \geq 0$. The function $T(r x)$ has a convergent Fourier series

$$
T(r x)=\sum_{m=-\infty}^{\infty} c_{m}^{\prime} e^{2 \pi i m x} \quad \text { for } 0<x<1 .
$$

Clearly

$$
c_{0}^{\prime}=\frac{1}{r} \sum_{l=0}^{r-1} T(l) ;
$$

and, for $m \neq 0$, by an argument used in Section 3 we have

$$
\begin{aligned}
c_{m}^{\prime} & =\int_{0}^{1} T(r x) e^{-2 \pi i m x} d x \\
& =\frac{1}{2 \pi i m}\left(\sum_{l=0}^{r-1} \chi\left(a g^{l}+b\right) e_{r}(-l m)-\sum_{l=0}^{r-1} \chi\left(a g^{l}+b\right)\right) \\
& =\frac{1}{2 \pi i m} \sum_{l=0}^{r-1} \chi\left(a g^{l}+b\right) e_{r}(-l m)+\frac{\chi(b)}{2 \pi i m} \\
& =\frac{1}{2 \pi i m r} \sum_{\psi} J(\chi, \bar{\chi} \psi) \psi(a) \chi \bar{\psi}(-b) \sum_{l=0}^{r-1} \psi\left(g^{l}\right) e_{r}(-l m)+\frac{\chi(b)}{2 \pi i m} \\
& =\frac{1}{2 \pi i m} J\left(\chi, \bar{\chi} \psi_{m}\right) \psi_{m}(a) \chi \bar{\psi}_{m}(-b)+\frac{\chi(b)}{2 \pi i m},
\end{aligned}
$$

where $\psi_{m} \in \widehat{F_{q}^{*}}$ being defined by $\psi_{m}(g)=e_{r}(s)$ with $0<s \leq r$ and $s \equiv m$ $(\bmod r)$.

Let $\chi$ be defined by $\chi(g)=e_{r}(t)$ with $0<t<r$. Then $\psi_{m} \neq \varepsilon, \chi$ if and only if $m \not \equiv 0, t(\bmod r)$. Thus, by (4.4) and (4.6) we get

$$
\left|c_{m}^{\prime}\right| \geq \frac{1}{2 \pi|m|}(\sqrt{q}-1) \quad \text { for } m \not \equiv 0, t(\bmod r) .
$$

Then, Theorem 4 follows from this, (4.5) and Parseval's formula as in Section 3 .

Acknowledgements. The author thanks the referee for his careful reading of the manuscript and suggestions on the revision of the paper. 


\section{References}

[1] D. A. Burgess, On character sums and primitive roots, Proc. London Math. Soc. (3) 12 (1962), 179-192.

[2] H. Davenport, Multiplicative Number Theory, 2nd ed., Springer, 1980.

[3] E. Dobrowolski and K. S. Williams, An upper bound for the sum $\sum_{n=a+1}^{a+H} f(n)$ for a certain class of functions $f$, Proc. Amer. Math. Soc. 114 (1992), 29-35.

[4] R. Lidl and H. Niederreiter, Finite Fields, Encyclopedia Math. Appl. 20, AddisonWesley, 1983.

[5] H. Niederreiter, On the distribution of pseudo-random numbers generated by the linear congruential method. III, Math. Comp. 30 (1976), 571-597.

[6] -, Quasi-Monte Carlo methods and pseudo-random numbers, Bull. Amer. Math. Soc. 84 (1978), 957-1041.

Department of Mathematics

University of Science and Technology of China

Hefei, 230026, Anhui

P.R. China

E-mail: yuhb@ustc.edu.cn

Received on 23.10.1998

and in revised form on 14.8.2000 